\title{
The Impact of the COVID-19 Pandemic on Stress and Other Psychological Factors in Pregnant Women Giving Birth During the First Wave of the Pandemic
}

\section{Theresa Hübner ( $\nabla$ huebner_t1@ukw.de)}

University Hospital Wurzburg Department of Obstetrics and Gynaecology: Universitatsklinikum Wurzburg Frauenklinik und Poliklinik https://orcid.org/0000-0003-2948-5315

\section{Tanja Wolfgang}

University Hospital Wurzburg: Universitatsklinikum Wurzburg

Ann-Catrin Theis

University Hospital Wurzburg Department of Obstetrics and Gynaecology: Universitatsklinikum Wurzburg Frauenklinik und Poliklinik

\section{Magdalena Steber}

University Hospital Wurzburg Department of Obstetrics and Gynaecology: Universitatsklinikum Wurzburg Frauenklinik und Poliklinik

\section{Lea Wiedenmann}

University Hospital Wurzburg Department of Obstetrics and Gynaecology: Universitatsklinikum Wurzburg Frauenklinik und Poliklinik

\section{Achim Wöckel}

University Hospital Wurzburg Department of Obstetrics and Gynaecology: Universitatsklinikum Wurzburg Frauenklinik und Poliklinik

\section{Joachim Diessner}

University Hospital Wurzburg Department of Obstetrics and Gynaecology: Universitatsklinikum Wurzburg Frauenklinik und Poliklinik

\section{Grit Hein}

University Hospital Wurzburg: Universitatsklinikum Wurzburg

\section{Marthe Gründahl}

University Hospital Wurzburg: Universitatsklinikum Wurzburg

\section{Ulrike Kämmerer}

University Hospital Wurzburg Department of Obstetrics and Gynaecology: Universitatsklinikum Wurzburg Frauenklinik und Poliklinik

\section{Sarah Kittel-Schneider}

University Hospital Wurzburg: Universitatsklinikum Wurzburg

\section{Catharina Bartmann}


University Hospital Wurzburg Department of Obstetrics and Gynaecology: Universitatsklinikum Wurzburg Frauenklinik und Poliklinik

\section{Research}

Keywords: COVID-19 pandemic, concern, depression, anxiety, maternal bonding and self-efficacy

Posted Date: December 7th, 2021

DOI: https://doi.org/10.21203/rs.3.rs-1113382/v1

License: (c) (1) This work is licensed under a Creative Commons Attribution 4.0 International License. Read Full License 


\section{Abstract}

\section{Background}

The onset of mental illness such as depression and anxiety disorders in pregnancy and postpartum period is common. The coronavirus induced disease 2019 (COVID-19) pandemic and the resulting public policy responses represent an exceptional situation worldwide and there are hints for adverse psychosocial impact, hence, the study of psychological effects of the pandemic in women during hospitalization for delivery and in the postpartum period is highly relevant.

\section{Methods}

Patients who gave birth during the first wave of the Corona pandemic in Germany (March to June 2020) at the Department of Obstetrics and Gynecology, University of Würzburg, Germany, were recruited at hospital admission for delivery. Biosamples were collected for analysis of SARS-CoV-2 infection and various stress hormones and interleukin-6 (IL-6). In addition to sociodemographic and medical obstetric data, survey questionnaires in relation to concerns about and fear of COVID-19, depression, stress, anxiety, loneliness, maternal self-efficacy and the mother-child bonding were administered at T1 (delivery stay) and T2 (3-6 month postpartum).

Results

In total, all 94 recruited patients had a moderate concern of severe acute respiratory syndrome coronavirus 2 (SARS-CoV2) at T1 with a significant rise at T2. This concern correlated with low to lowmedium general psychosocial stress levels and stress symptoms, and the women showed a significant increase of active coping from T1 to T2. Anxiety levels were low and the Edinburgh Postnatal Depression Scale showed a medium score of 5 with a significant (T1), but only week correlation with the concerns about SARS-CoV-2. In contrast to the overall good maternal bonding without correlation to SARS-CoV-2 concern, the maternal self-efficiency correlated negatively with the obstetric impairment caused by the COVID-19 pandemic.

Conclusion

Obstetrical patients ' concerns regarding SARS-CoV-2 and the accompanying pandemic increased during the course of the pandemic correlating positively with stress and depression. Of note is the increase in active coping over time and the overall good mother-child-bonding. Maternal self-efficacy was affected in part by the restrictions of the pandemic.

Clinical trial registration: DRKS00022506

\section{Plain English Summary}


The global pandemic of COVID-19 (coronavirus induced disease 2019) is challenging our society in many ways. Especially pregnant women are facing extraordinary conditions and worries, like uncertain risks for mother and fetus in case of infection, restricted prenatal classes or restricted visitor regulations in hospitals. Particularly it is known that pregnancy and the postnatal period are presenting a more psychologically vulnerable time in a woman's life.

Developing the GeZeCO study, we aimed to focus on the pandemic's effects on mental health of pregnant women during this time. Women giving birth in the department of obstetrics of the University Hospital Würzburg were asked to participate in the study. In total, 94 women completed several questionnaires concerning their mental health postpartum and again after three to six months. Further, we took blood samples of the women during the delivery stay and registered sociodemographic and obstetrical data.

Our results showed, that the women's concern relating to COVID-19, as well as the level of depression and anxiety raised during the pandemic. In addition, the self-efficacy of the mothers was affected by the restriction measures. Despite this, the women had at large a good mother-child-bonding and their competence of active coping increased during time.

In summary, we did find that the mental health of obstetrical patients is impaired by the COVID-19 pandemic. This points out the importance of not only attending to physical health but also taking care of psychological stress and mental health problems of obstetrical patients during this exceptional time.

\section{Introduction}

In March 2020 the coronavirus induced disease 2019 (COVID-19) was declared a worldwide pandemic by the World Health Organization (WHO) [1] and confronts global society with new and unexpected challenges. The effects of this pandemic, which arrived in Germany in March 2020, do not only refer to the infection with severe acute respiratory syndrome coronavirus 2 (SARS-CoV-2) and its possible short and long-term consequences, but also death from COVID-19. Infection control measures including lockdown regulations affect the everyday life of each individual in various ways. In addition to wearing face masks and general hygiene requirements, this includes social contact restrictions, restriction of the range of movement, short-time work and / or the closure of workplaces, increased work-from-home arrangements as far as possible, restriction or closure of schools and kindergartens, restriction of shopping opportunities as well as quarantine measures in case of COVID-19 disease [2]. In the medical field, in addition to strict hygiene measures at the beginning of the pandemic, examinations, treatments and operations that were not absolutely urgent were postponed in order to keep the highest possible number of intensive care beds available [3]. There was also a ban or at least restriction on hospital visitors.

Such situations due to an infectious pathogenic coronavirus strain were known to a more moderate extent from the severe acute respiratory syndrome (SARS) pandemic from 2002 until 2003 and the Middle East respiratory syndrome (MERS) epidemic of 2015 [4]. Lessons of these past pandemics 
showed that in the absence of vaccines or proven effective therapies prevention is the key to disrupt the chain of infections [5].

It is known that pregnancy and the postpartum time present one of the most mentally vulnerable periods in a woman's life with a higher risk for the onset of depression and anxiety [6, 7]. In addition, maternal mental illness in the postpartum period can negatively affect the development of the child and therefore needs to be diagnosed and treated urgently and sufficiently [8]. Thus, the cohort of women pregnant during the pandemic seems to be a group of particular interest and concern regarding the possible effects of pandemic restrictions on mental health [9]. Based on experiences from disasters like earthquakes, hurricanes or terrorist attacks the exposure to disaster and associated stress can lead to an impairment of mental health of pregnant women and can have an impact on the pregnancy outcome [10]. Analogies to a global pandemic are to be expected.

At the beginning of the COVID-19 pandemic, no prior knowledge was available as to whether a maternal SARS-CoV-2 infection could be transmitted via the placenta to the fetus and what effects COVID-19 would have on the fetus and the course of pregnancy. Whereas Middle East respiratory syndrome coronavirus (MERS-CoV) and severe acute respiratory syndrome coronavirus (SARS-CoV) were found to be associated with adverse pregnancy outcomes as miscarriage or fetal death, preterm birth and a higher risk for severe maternal illness $[11,12]$ the current data published so far fails to prove any clear evidence for vertical transmission of SARS-CoV-2 to the fetus or newborn while the findings concerning a higher maternal risk for severe infection remain ambiguous $[11,13,14]$. Nevertheless, a Canadian study showed several changes in perinatal outcomes during the pandemic, for example a higher rate of obstetric intervention in early pregnancy [15]. Further, meta-analyses supported a higher risk of severe course of COVID-19 pregnancy and adverse pregnancy outcomes [16, 17]. An increasing prevalence for mental health problems like anxiety and depression of mothers and pregnant women was reported in previous studies $[14,18]$.

During the lockdown measures, there were also strict visitor regulations in German obstetric departments, hospital wards and delivery rooms. A higher stress level and prevalence of anxiety for pregnant women were shown at the onset of the pandemic and restrictive measures in previous studies [19-21].

The aim of the GeZeCO study was to detect the psychological effects of the COVID-19 pandemic in patients during their delivery stay and in the postpartum period in Germany. Therefore, we focused entirely on the effects of the pandemic on depression, stress and anxiety during course of time and as first examined the maternal self-efficacy and the mother-child bonding.

\section{Methods}

Study population

Women who have given birth during the first wave of the COVID-19 pandemic in Germany (March to June 2020) at the Department of Obstetrics and Gynecology, University Hospital of Würzburg, were asked to 
take part in this study. Details of the entire study procedure, the inclusion criteria and an overview of the questionnaires and the bio samples are shown in Figure 1. The study was approved by the ethics committee of the University of Würzburg (No. 70/20 Amendment). After receiving verbal and written information, the patients agreed to participate in the study with written informed consent. The study adhered to the Declaration of Helsinki, version 2013.

\section{Laboratory analysis}

Biosamples were collected for analysis of SARS-CoV-2 infection and various stress hormones and interleukin-6 (IL-6). Throat swabs were examined for the presence of SARS-CoV-2 at the Institute of Virology at the University of Würzburg or in the research lab of the Department of Gynecology using reverse transcriptase quantitative polymerase chain reaction (RT-qPCR) or reverse transcriptase polymerase chain reaction (RT-PCR) respectively. Enzyme-linked immunosorbent assays (ELISA) were performed with frozen blood serum samples according to manufacturer's instructions in the research lab of the Department of Gynecology to test the immunoglobulins G and M of SARS-CoV-2 (EL-2006-9601 G, EL-2606-9601-2M, Euroimmun, Lübeck, Germany), cortisol (ARG81162, arigo biolab), adrenaline (E-EL0045, Elbscience), norepinephrine (E-EL-0047, Elbscience), dopamine (E-EL-0046, Elbscience) and IL-6 (DY206-05, R\&D).

COVID-19 pandemic questionnaire (CPQ) and Fear of Covid-19 Scale (FCV-19S)

The first part of the self-designed COVID-19 pandemic questionnaire asked for specific symptoms of the diseases during pregnancy in an open-ended question and then for following symptoms; fever (temperature higher than $38.5^{\circ} \mathrm{C}$ ), cough, shortness of breath, muscle and joint pain, sore throat, headache, nausea/vomiting, nasal congestion, diarrhea, taste and/or smell problems and pneumonia. Finally, a possible contact with a SARS-CoV-2 positive person, a historical positive throat swab for SARSCoV-2 and / or a COVID19 disease were queried. The questions of the second part are shown in Table 1 and are divided into the concern scale (questions 1-4), the concern over time scale (questions 5-8) and the impairment scale (questions 9-11). The results of the scales were calculated as the sum of the appropriate questions. A Likert scale was applied for questions 1-8 $(1=$ No, never; $2=1$ have thought about it, but was not concerned; $3=\mathrm{I}$ am a little concerned; $4=\mathrm{I}$ am often concerned; $5=\mathrm{I}$ am concerned about it all the time) and questions 9-11 ( $1=$ not at all; 2 = a little bit, $3=$ moderately; 4 = quite a lot; 5 = a lot). The Fear of Covid-19 scale (FCV-19S) was published by Ahorsu and coworkers and the sum score of all questions was used to measure the fear of COVID-19 [22].

Stress and coping inventory (SCl)

The stress and coping inventory ( $\mathrm{SCl})$ is a German-language stress questionnaire with 54 items [23]. The first 21 items of the $\mathrm{SCl}$ are divided into three subscales consisting of seven items each: "stress caused by insecurity", "stress caused by being overwhelmed" and "stress caused by loss". Here, a seven-point Likert scale from "not burdened" to "very heavily burdened" is used. Together, the three subscales assess the total stress level. Here, a seven-point Likert scale from "not burdened" to "very heavily burdened" is 
used. The following 13 items measure physical stress symptoms on a four-point Likert scale ("does not apply at all", "applies a bit", "moderately applies" and "applies completely"). The same Likert scale is applied to the last 20 items evaluating coping strategies. The coping items can be divided into "positive coping", "active coping", "coping by support", "coping by believing in God or powers that be" and "coping by drinking alcohol and/or smoking", each with four items. For the evaluation of SCl scales, the sum of all item points of each scale was formed following the instructions of the evaluation manual [24].

Depression and anxiety (EPDS and ASI-3)

The Edinburgh Postnatal Depression Scale (EPDS) was developed in 1987 as a screening instrument for postnatal depression [25] and translated and adapted to German [26-28]. The total score is the sum of all ten items with a four-point Likert scale (from 0 to 3). An EPDS value of 10 or higher has a middle to high probability for depression [29].

The Anxiety Sensitivity Index-3 (ASI-3) was developed by Taylor in 2007 [30] and translated as well as adapted to German by Kemper [31]. The response of the 18 items with a five-point Likert scale were summed up in a total score.

Maternal self-confidence (LMSCS) and parental bonding (PBQ)

The Lips maternal self-confidence scale (LMSCS) was developed by Bloom and Lips in 1993 and translated to German [32]. It contains 25 questions with a six point Likert scale. The total score is the sum of each item.

The Parental Bonding Questionnaire (PBQ) is a self-reported questionnaire with 25 items. The answers of its six-point Likert scale have been added up to a total score [33,34].

The Loneliness and Isolation during Social Distancing Scale (LISD Scale)

The Loneliness and Isolation during Social Distancing (LISD) Scale questionnaire was developed and validated by Gründahl and coworkers on the occasion of the COVID-19 pandemic [35]. This questionnaire assesses loneliness and isolation in the context of social distancing on a state and trait level. The first part with 12 items concerns a person's current experience and feelings (state) and is divided into two subscales ("Ionely and isolated" and "connected and supported"). The second part of the LISD scale consists of 13 items and assesses a person's experience and feelings in general (trait). It is divided into three subscales ("loneliness and isolation", "sociability and sense of belonging" and "social support and closeness"). For each item, a five point Likert scale is used (ranging from "strongly agree" to "strongly disagree") [35].

\section{Statistical analysis}

The software SPSS Statistics 26 (IBM) was used to perform statistical analyses and create tables. For normal distributions, Shapiro-Wilk tests were performed. Data are presented accordingly as mean $( \pm$ 
standard deviation [SD]) or median (interquartile percentile) values. Wilcoxon test and Mann-Whitney-Utest were used to detect significant differences in non-parametric data. P-values $\leq 0.05$ were rated as statistically significant. Cronbach's alpha was determined for the score reliability and Spearman's rho test for inter-scale correlation.

\section{Results}

Study population

A total of 94 patients were enrolled in the study from April to June 2020 (T1). Basic characteristics and obstetric information of the study population are shown in Table 2. 62 patients (66.0\%) participated in the follow-up at 3 to 6 months (T2) after birth (September to November 2020).

Infection status

None of the patients had knowingly suffered from COVID-19 and none were tested positive for SARS-CoV2 by RT-qPCR or RT-PCR. 16 (17.0\%) patients reported no respiratory infection during pregnancy, but 70 patients (74.5\%) specified one or more of the following symptoms: 6 (6.4\%) fever, $23(24.5 \%)$ cough, 26 (27.7\%) shortness of breath, 21 (22.3\%) muscle and joint aches, $28(29.8 \%)$ headache, $28(29.8 \%)$ throat sore, 27 (28.7\%) nausea and vomiting, 18 (19.1\%) diarrhea, 47 (50.0\%) congested nose, 8 (8.5\%) new loss of taste and smell, none ( $0 \%$ ) pneumonia. No patients had positive IgM antibodies against SARS-CoV-2, but two patients had positive IgG antibodies. The first of those two patients reported symptoms like shortness of breath, muscle and joint aches, and congested nose during the course of pregnancy. The second patient suffered from headache, nausea and vomiting, and diarrhea during pregnancy. Both patients denied infectious diseases during pregnancy and attributed symptoms to concomitant diseases and/or pregnancy problems.

\section{Concerns of COVID-19}

Table 1 presents the results of the COVID-19 pandemic questionnaire answered by 94 obstetric patients at T1. The median of all questions was either 2.0 or 3.0 meaning "thoughts/a little concern" in the concern scale and the concern over time scale as well as "a little bit/moderately" in the impairment scale. Altogether, the median of the concern scale remained the same with 9.00 (8.00-12.00) at T1 and 9.00 (8.00-12.00) at T2 ( $p=0.663)$. In contrast to that, the values of the concern over the time scale $(p=0.007)$, the impairment scale $(p=0.003)$ and the overall concern scale (sum of the concern scale, the concern over time scale and the impairment scale; $p=0.004$; Wilcoxon test) increased significantly. The concern over time scale counted 8.00 (7.00-11.00) at T1 and 8.50 (7.00-11.00) at T2, the impairment scale 8.00 (7.00$11.00)$ at T1 and 9.00 (7.00-11.00) at T2, the overall concern scale $26.00(22.00-32.00)$ at T1 and 28.00 (23.00-32.00) at T2. Cronbach's alpha at T1 and T2 was 0.890/0.814 in the concern scale, $0.873 / 0.840$ in the concern over time scale, $0.777 / 0.782$ in the impairment scale and $0.867 / 0.838$ of the overall concern scale (sum of the concern scale, the concern over time scale and the impairment scale). 
The median of the Fear of COVID-19 scale (FCV-19S) at T2 was 12.00 (11.00-16.00) with a total score of 35 points representing maximum fear. Cronbach's alpha was 0.758 . The results of the FCV-19S correlated significantly with the overall concern scale of the COVID-19 pandemic questionnaire at T1 $\left(r_{s}=0.283 ; p=\right.$ $0.030)$ and the overall concern scale of the COVID-19 pandemic questionnaire at T2 $\left(r_{s}=0.405 ; p=\right.$ 0.001). Furthermore, there were significantly positive correlations between FCV-19S and the following subscales of the COVID-19 pandemic questionnaire: Concern over time scale at T1 $\left(r_{s}=0.388 ; p=0.002\right)$ and at $\mathrm{T} 2\left(r_{s}=0.381 ; p=0.003\right)$ and concern scale at $\mathrm{T} 2\left(r_{s}=0.357 ; p=0.005\right)$.

\section{Stress and Coping Inventory}

The results of the different scales of the $\mathrm{SCl}$ were summarized in Table 3 representing rather low to lowmedium stress levels and stress symptoms. Coping by believing in God or powers had a medium value and coping by drinking alcohol and/or smoking was scarcely present in our sample. The coping scales "positive coping", "active coping" and "coping by support" had rather higher or high values with a significant increase of active coping from T1 to T2. Cronbach's alpha at T1 and T2 was higher (in the most cases even clearly higher) than 0.600 in the different scales except for the coping by drinking alcohol and/or smoking scale with a Cronbach's alpha of 0.528. The total load of stress of SCI at T1 correlated significantly with the overall concern scale of the COVID-19 pandemic questionnaire at T1 $\left(r_{s}=\right.$ $0.284 ; p=0.008$ ) as well as the subscales at T1 shown in Table 4 .

There was no significant correlation between the total load of stress measured by the SCl at T2 and the overall concern scale of the COVID-19 pandemic questionnaire at T2 $\left(r_{s}=0.047 ; p=0.723\right)$. By comparison with FCV-19S, there were no significant correlations between stress or stress symptoms and FCV-19S. Only positive coping at T2 showed a significant negative correlation to FCV-19S $\left(r_{s}=-0.257\right.$, $\mathrm{p}=0.046)$.

\section{Depression and Anxiety}

The median score of the EPDS measuring depression at T1 was 5.00 (2.00-7.00) with a insignificant small decrease at T2 (4.00 [2.00-7.00]; $p=0.312)$. Cronbach's alpha was 0.772 at T1 and 0.843 at T2. $10.64 \%$ (10 women) had an EPDS value of 10 or higher. The score of the EPDS at T1 correlated significantly with the overall concern scale of the COVID-19 pandemic questionnaire $\left(r_{s}=0.253 ; p=0.02\right)$. This significant correlation was absent at $\mathrm{T} 2\left(\mathrm{r}_{\mathrm{s}}=0.11 ; \mathrm{p}=0.41\right)$. There were also significant positive correlations between EPDS at T1 and the subscales concern over time at T1 $\left(r_{s}=0.212 ; p=0.044\right)$ and impairment scale at T1 $\left(r_{s}=0.240 ; p=0.022\right)$. The other subscales showed no correlation. Furthermore, there were no significant correlations of EPDS at T1 or T2 with FCV-19S. The median trait score of the ASI-3 was 11.50 (6.00-19.00; Cronbach's alpha 0.926) and indicated a rather low level of anxiety in view of an overall possible score of 72 . The results of the COVID-19 pandemic questionnaire (concern scale, concern over time scale, impairment scale and overall concern scale) did not correlate significantly with the results of the ASI-3. In contrast, there was a significant correlation between the FCV-19S and the ASI-3 $\left(r_{s}=0.299, p=0.026\right)$. 
The median of the PBQ was 6.00 (3.00-9.00; Cronbach's alpha 0.790) representing good maternal bonding. There were no significant correlations with the COVID-19 pandemic questionnaire and the FCV19S. The median of the LMSCS was 119 (110-124; Cronbach's alpha 0.829) and showed a rather high self-confidence with a maximum of 144 . There were no correlations to both COVID-19 questionnaires with one exception: The impairment scale of the COVID-19 pandemic questionnaire correlated negatively with the LMSCS $\left(r_{s}=-0.309, p=0.016\right)$.

Loneliness and Isolation during Social Distancing

The LISD Scale hardly showed loneliness and isolation, as well as high social support in the trait and state scales. On all scales, the maximum score was 5.00. In detail, the mean score of state factor 1 (lonely and isolated) was 2.00 (1.67-2.67) and the mean state factor 2 score (connected and supported) was 4.67 (4.33-5.00). The mean trait factor 1 score (loneliness and isolation) was 1.50 (1.25-1.75), the mean trait factor 2 score (sociability and sense of belonging) was 4.20 (3.80-4.40), and the mean trait factor 3 score (social support and closeness) was 4.75 (4.50-5.00). Cronbach's alpha was 0.172 for the state factor 2 and 0.415 for trait factor 3 . In the other scales, it was $>0.600$. Table 5 presents possible correlations between the LISD Scale and the CPQ as well as the FVC-19S.

Biomarker

Table 6 shows the correlation of stress hormones (cortisol, adrenaline, norepinephrine, dopamine) as well as the infection parameter IL- 6 with the results of the questionnaires at T1. We found a negative correlation between the dopamine level in the periperial blood and the total load of stress $\left(r_{s}=-0.288\right.$, $p=0.007)$, stress symptoms $\left(r_{s}=-0.231, p=0.032\right)$ as well as the overall concern scale in the COVID-19 pandemic questionnaire $\left(r_{s}=-0.212, p=0.047\right)$. Further, the levels of IL-6 correlated negatively with the total load of stress $\left(r_{s}=-0.227, p=0.034\right)$.

\section{Discussion}

Worldwide research is currently focused on SARS-CoV-2, possible therapeutic targets and preventive measures, as well as the COVID-19 pandemic and its various potential impacts on health care, mental health and economic effects. The majority of studies center on physical effects and immunologic pathways of infection. Pregnant women were particularly observed regarding vertical transmission, fetal infection and pregnancy outcome during the first wave of the pandemic, while their mental health was not investigated [36]. As previous results show a general increase of mental health problems, women, especially in perinatal situations, seem to have a higher risk for impairment of mental health [37-39] or loneliness and isolation [40-42]. For this reason, the present study aimed to fill this gap in current knowledge. 
In our study, the scores of the COVID-19 pandemic questionnaire showed overall a low to moderate overall level of concern, whereas the level of concern increased over the course of the pandemic, i.e., between delivery stay (T1) and three to six months postpartum (T2). This corresponds to findings in other studies [43], although most of studies show results from one date and no comparison of stress level to a later date during the ongoing pandemic [43-47].

The $\mathrm{SCl}$ results showed low stress levels, while a significant increase in active coping mechanisms could be detected between delivery stay to postpartum assessment (from T1 to T2), especially in the following subgroups: women under 35 years, academics, body mass index $(\mathrm{BMI})<25 \mathrm{~kg} / \mathrm{m}^{2}$, caesarian section, first birth and high-risk pregnancies. Coping strategies are known to be an important factor for maternal mental health [48-50]. Our results support the hypothesis of an Italian longitudinal study where women are likely to develop better resilience in the course of the pandemic [51].

At the delivery stay (T1), higher total load of stress related to more overall COVID-19-related concern, especially stress by insecurity and stress by being overwhelmed. This effect dissolves over time and cannot be detected anymore at the postpartum assessment (T2), which might be interpreted as an effect of habituation and of the improvement of coping strategies during the pandemic as described above [52-54].

No significant correlations between psychosocial stress or stress symptoms of SCl and FCV-19S could be shown, considering the fact, that the FCV-19S focusses on general fear concerning COVID-19 and not specific worries of pregnant women during the pandemic as the CPQ [22]. A possible reason to explain the lack of relationships could be that the stressors for our group are the specific concerns relating to pregnancy and the obstetrical situation and not a generally raised level of fear. In contrast to our observed low score of the FCV-19S, another study reported high levels [55]. This difference could be due to differences in ethnic and socio-demographic aspects in the tested population. It is also possible that the time period studied during the COVID-19 pandemic, the week of pregnancy and the care capacity of the health care system are important confounding factors.

Many studies reported increased depression and anxiety symptoms in pregnant women during the COVID-19 pandemic [42,56-58]. In our study, we have found relatively low scores of the EPDS and the ASI-3. The rate of about $10 \%$ estimated depression detected by the EPDS was in the normal prevalence range (about 7-13\%) in pregnancy and postpartum $[6,59]$. At first this may seem to contradict the previously mentioned studies $[42,57,58,60]$. However, due to our study design (Figure 1 ) we were unable to compare to pre-pandemic values. Instead, we investigated relationships of depression and COVID-19 specific factors. More COVID-19 related concerns related to higher depression, but not anxiety scores. In contrast, COVID-19 related fear correlated positively to anxiety, but not depression. There seems to be a difference between obstetric concerns in the face of COVID-19 pandemic and general fear of COVID-19, although they correlated to some degree. 
In the face of the SARS-CoV-1 outbreak, Lee and coworkers have shown that anxiety and depression did not increase compared to pre-outbreak levels. The authors attribute this to increased social support [61]. This might also be the underlying reason for this study sample's low scores in anxiety and depression. Social support during pregnancy does not only have an influence on mental health symptoms such as depression but also on pregnancy outcome [62]. Although some studies report low social support and the consequent negative impact on women in late pregnancy and postpartum during the COVID-19 pandemic $[63,64]$, pregnant women may also have benefited from increased flexibility of work schedules and jobs due to COVID-19 pandemic restrictions, such as themselves or their husbands' ability to work from home. Strengthening this line of evidence, our results show low to low-medium loneliness and social isolation and high social support on both the state and the trait level. Being lonely and socially isolated (state and trait) was associated with higher impairment scores both during the delivery stay and several months postpartum, while being socially connected and supported (state) related to lower impairment. Note that interpretation of the indicators of social support are limited by a low reliability. This may have been caused by extremely skewed score distributions and high scores for being connected and supported (state) and for social support and connectedness (trait 3). Nonetheless, these findings emphasize the effect of social support and particularly isolation and loneliness on the level of mental health impairment.

Although many factors influence the mother-infant-bonding with stress levels caused by the COVID-19 pandemic potentially being among them $[65,66]$, we detected a good maternal-infant bonding in our study, independent of concerns and fears regarding SARS-CoV-2 and the COVID-19 pandemic. This also corresponds well with the observed low stress levels. However, the impairment by the COVID-19 pandemic correlated negatively with maternal self-confidence. This was also the finding of Vazquez-Vazquez and co-authors. They suggest that maternal lack of contact with other mothers through the restrictions caused by the COVID-19 pandemic had an impact on the assessment of maternal self-efficacy [67].

Here, we found a significant inverse relationship of peripheral dopamine level with level of stress and level of concern in the COVID-19 pandemic questionnaire. There were also similar results for the correlation between peripheral dopamine levels and depression scores. These findings support the hypothesis that dopamine plays an important role in modulating stress-coping mechanisms although most of studies focus on dopamine levels in the central nervous system and not peripheral levels [6872].

Our study showed a correlation of IL-6 and elevated stress levels, which emphasize the findings of other authors, that higher levels of IL- 6 are associated with mental health problems such as depression and anxiety [73-75].

For serum levels of cortisol, adrenaline and noradrenaline we did not find any significant correlation. The results of other studies are heterogeneous, levels of these hormones are shown to be strongly dependent on individual baselines and circadian rhythm as well as the time interval to stressors and intensity of stressors, which our study did not take into account $[76,77]$. 
A limitation of our study was that there were no clearly defined survey time or conditions for the survey as well as the maternal blood sample, which owed to the course of labour and the workflow in delivery rooms. This may have lead to a blur in the obtained data, however we rate this to be of minor influence on our overall finding.

\section{Conclusion}

In our study we found increased levels of concern of obstetric patients regarding the COVID-19 pandemic during the course of the pandemic in Germany that correlates particularly with stress and depression levels. Our results suggest that raised levels of stress are rather of situational nature and not a result of generally raised levels of fear and depression. The women of our study population experienced a raised level of active coping over time as well as good levels of parental bonding, neither being adversely affected by measured impairment of the pandemic. In contrast, maternal self-efficacy was influenced in part by the restrictions imposed by the pandemic.

Our findings support previous work studying the psychological effects of the pandemic and an increased risk for mental health problems. They emphasize the special situation of pregnant women during this period and as well the need for monitoring, prevention and intervention.

\section{Abbreviations}

ASI-3: Anxiety Sensitivity Index-3

BMI: body mass index

COVID-19: coronavirus induced disease 2019

CPQ: COVID-19 pandemic questionnaire

ELISA: enzyme-linked immunosorbent assays

EPDS: Edinburgh Postnatal Depression Scale

FCV-19S: Fear of Covid-19 Scale

IL-6: interleukin-6

LISD: Loneliness and Isolation during Social Distancing Scale

LMSCS: Lips maternal self-confidence scale

MERS: Middle East respiratory syndrome

MERS-CoV: Middle East respiratory syndrome coronavirus 
PBQ: Parental Bonding Questionnaire

RT-PCR: reverse transcriptase polymerase chain reaction

RT-qPCR: reverse transcriptase quantitative polymerase chain reaction

SARS: severe acute respiratory syndrome

SARS-CoV: severe acute respiratory syndrome coronavirus

SARS-CoV-2: severe acute respiratory syndrome coronavirus 2

SCl: stress and coping inventory

SD: standard deviation

WHO: World Health Organization

\section{Declarations}

Ethics approval and consent to participate

The study was approved by the ethics committee of the University of Würzburg (No. 70/20 Amendment). After verbal and written information, the patients agreed to participate in the study with written informed consent. The study adhered to the Declaration of Helsinki, version 2013.

Consent for publication

Not applicable.

Availability of data and material

The datasets generated and/or analysed during the current study are available from the corresponding author on reasonable request.

Competing interests

The authors declare that they have no competing interests.

Funding

Not applicable.

Authors' contributions

Data Analysis, Statistics and Manuscript Design: TH, CB, TW, ACT, 
Project Design and Data Collection: TH, CB, ACT, MS, LW, UK, AW, GH, MG, SKS, JD Conception and Study Design: TH, CB, UK, SKS

All authors read and approved the final manuscript.

Acknowledgements

We, the authors, would like to thank Michaela Kapp for her excellent technical assistance, Niclas Moneke for many helpful comments, Peter Heuschmann, Anna Grau and Stephanie Stangl for providing support in data management and our midwifes for their support every day and night in the delivery rooms.

\section{References}

1. Cucinotta D, Vanelli M. WHO Declares COVID-19 a Pandemic. Acta Biomed. 2020;91(1):157160.10.23750/abm.v91i1.9397

2. Khalifa SAM, Swilam MM, El-Wahed AAA, Du M, El-Seedi HHR, Kai G, Masry SHD, Abdel-Daim MM, Zou X, Halabi MF, Alsharif SM, El-Seedi HR. Beyond the Pandemic: COVID-19 Pandemic Changed the Face of Life. Int J Environ Res Public Health. 2021;18(11).10.3390/ijerph18115645

3. Søreide K, Hallet J, Matthews JB, Schnitzbauer AA, Line PD, Lai PBS, Otero J, Callegaro D, Warner SG, Baxter NN, Teh CSC, Ng-Kamstra J, Meara JG, Hagander L, Lorenzon L. Immediate and long-term impact of the COVID-19 pandemic on delivery of surgical services. Br J Surg. 2020;107(10):1250$1261.10 .1002 /$ bjs. 11670

4. de Wit E, van Doremalen N, Falzarano D, Munster VJ. SARS and MERS: recent insights into emerging coronaviruses. Nat Rev Microbiol. 2016;14(8):523-534.10.1038/nrmicro.2016.81

5. Munnoli PM, Nabapure S, Yeshavanth G. Post-COVID-19 precautions based on lessons learned from past pandemics: a review. Z Gesundh Wiss. 2020:1-9.10.1007/s10389-020-01371-3

6. Bennett HA, Einarson A, Taddio A, Koren G, Einarson TR. Prevalence of depression during pregnancy: systematic review. Obstet Gynecol. 2004;103(4):698-709.10.1097/01.AOG.0000116689.75396.5f

7. Becker M, Weinberger T, Chandy A, Schmukler S. Depression During Pregnancy and Postpartum. Curr Psychiatry Rep. 2016;18(3):32.10.1007/s11920-016-0664-7

8. Gunlicks ML, Weissman MM. Change in child psychopathology with improvement in parental depression: a systematic review. J Am Acad Child Adolesc Psychiatry. 2008;47(4):379389.10.1097/CHI.0b013e3181640805

9. Caparros-Gonzalez RA, Alderdice F. The COVID-19 pandemic and perinatal mental health. J Reprod Infant Psychol. 2020;38(3):223-225.10.1080/02646838.2020.1786910

10. Harville E, Xiong X, Buekens P. Disasters and perinatal health:a systematic review. Obstet Gynecol Surv. 2010;65(11):713-728.10.1097/OGX.0b013e31820eddbe

11. Wang CL, Liu YY, Wu CH, Wang CY, Wang CH, Long CY. Impact of COVID-19 on Pregnancy. Int J Med Sci. 2021;18(3):763-767.10.7150/ijms.49923 
12. Schwartz DA, Graham AL. Potential Maternal and Infant Outcomes from (Wuhan) Coronavirus 2019nCoV Infecting Pregnant Women: Lessons from SARS, MERS, and Other Human Coronavirus Infections. Viruses. 2020;12(2).10.3390/v12020194

13. Zlochiver V, Tilkens B, Perez Moreno AC, Aziz F, Jan MF. COVID-19 Deliveries: Maternal Features and Neonatal Outcomes. J Patient Cent Res Rev. 2021;8(3):286-289.10.17294/2330-0698.1848

14. Kotlar B, Gerson E, Petrillo S, Langer A, Tiemeier H. The impact of the COVID-19 pandemic on maternal and perinatal health: a scoping review. Reprod Health. 2021;18(1):10.10.1186/s12978-02101070-6

15. Liu S, Dzakpasu S, Nelson C, Wei SQ, Little J, Scott H, Joseph KS. Pregnancy outcomes during the COVID-19 pandemic in Canada, March to August 2020. J Obstet Gynaecol Can.

2021.10.1016/j.jogc.2021.06.014

16. Wei SQ, Bilodeau-Bertrand M, Liu S, Auger N. The impact of COVID-19 on pregnancy outcomes: a systematic review and meta-analysis. Cmaj. 2021;193(16):E540-e548.10.1503/cmaj.202604

17. Allotey J, Stallings E, Bonet M, Yap M, Chatterjee S, Kew T, Debenham L, Llavall AC, Dixit A, Zhou D, Balaji R, Lee SI, Qiu X, Yuan M, Coomar D, Sheikh J, Lawson H, Ansari K, van Wely M, van Leeuwen E, Kostova E, Kunst H, Khalil A, Tiberi S, Brizuela V, Broutet N, Kara E, Kim CR, Thorson A, Oladapo OT, Mofenson L, Zamora J, Thangaratinam S. Clinical manifestations, risk factors, and maternal and perinatal outcomes of coronavirus disease 2019 in pregnancy: living systematic review and metaanalysis. Bmj. 2020;370:m3320.10.1136/bmj.m3320

18. Koyucu RG, Karaca PP. The Covid 19 outbreak: Maternal Mental Health and Associated Factors. Midwifery. 2021;99:103013.10.1016/j.midw.2021.103013

19. Omowale SS, Casas A, Lai YH, Sanders SA, Hill AV, Wallace ML, Rathbun SL, Gary-Webb TL, Burke LE, Davis EM, Mendez DD. Trends in stress among pregnant and postpartum women during the COVID19 pandemic. JMIR Ment Health. 2021.10.2196/30422

20. Nowacka U, Kozlowski S, Januszewski M, Sierdzinski J, Jakimiuk A, Issat T. COVID-19 PandemicRelated Anxiety in Pregnant Women. Int J Environ Res Public Health. 2021;18(14).10.3390/ijerph18147221

21. Morniroli D, Consales A, Colombo L, Bezze EN, Zanotta L, Plevani L, Fumagalli M, Mosca F, Giannì ML. Exploring the Impact of Restricted Partners' Visiting Policies on Non-Infected Mothers' Mental Health and Breastfeeding Rates during the COVID-19 Pandemic. Int J Environ Res Public Health. 2021;18(12).10.3390/ijerph18126347

22. Ahorsu DK, Lin CY, Imani V, Saffari M, Griffiths MD, Pakpour AH. The Fear of COVID-19 Scale: Development and Initial Validation. Int J Ment Health Addict. 2020:1-9.10.1007/s11469-020-00270-8

23. Satow, L. (2012). Stress- und Coping-Inventar (SCI): Vollständige Test- und Skalendokumentation. verfügbar unter: http://www.drsatow.de/tests/stress-und-coping-inventar/

24. Satow, L. (2012). SCl - Stress- und Coping-Inventar [Fragebogen mit Beispielen und Skalendokumentation]. In Leibniz-Zentrum für Psychologische Information und Dokumentation 
(ZPID) (Hrsg.), Elektronisches Testarchiv (PSYNDEX Tests-Nr. 9006508). Trier: ZPID. https://doi.org/10.23668/psycharchives.424.

25. Cox JL, Holden JM, Sagovsky R. Detection of postnatal depression. Development of the 10-item Edinburgh Postnatal Depression Scale. Br J Psychiatry. 1987;150:782-786.10.1192/bjp.150.6.782

26. Muzik M, Klier CM, Rosenblum KL, Holzinger A, Umek W, Katschnig H. Are commonly used self-report inventories suitable for screening postpartum depression and anxiety disorders? Acta Psychiatr Scand. 2000;102(1):71-73.10.1034/j.1600-0447.2000.102001071.x

27. Bergant AM, Nguyen $T$, Heim $K$, Ulmer $H$, Dapunt $O$. [German language version and validation of the Edinburgh postnatal depression scale]. Dtsch Med Wochenschr. 1998;123(3):35-40.10.1055/s-20071023895

28. Deutsche Version als Befindlichkeitsbogen“: Herz, E. (1996). Befindlichkeitsbogen. Deutsche Version des Edinburgh Postnatal Depression Scale von Cox, J. L., Holden, J. M. \& Sagowski, R. (1987).

29. Levis B, Negeri Z, Sun Y, Benedetti A, Thombs BD. Accuracy of the Edinburgh Postnatal Depression Scale (EPDS) for screening to detect major depression among pregnant and postpartum women: systematic review and meta-analysis of individual participant data. Bmj. 2020;371:m4022.10.1136/bmj.m4022

30. Taylor S, Zvolensky MJ, Cox BJ, Deacon B, Heimberg RG, Ledley DR, Abramowitz JS, Holaway RM, Sandin B, Stewart SH, Coles M, Eng W, Daly ES, Arrindell WA, Bouvard M, Cardenas SJ. Robust dimensions of anxiety sensitivity: development and initial validation of the Anxiety Sensitivity Index3. Psychol Assess. 2007;19(2):176-188.10.1037/1040-3590.19.2.176

31. Kemper CJ, Ziegler, M. \& Taylor, S. ASI-3. Angstsensitivitätsindex-3 [Verfahrensdokumentation und Fragebogen]. In Leibniz-Institut für Psychologie (ZPID) (Hrsg.), Open Test Archive. Trier: ZPID. 2011.https://doi.org/10.23668/psycharchives.4526

32. Reck C, Noe D, Gerstenlauer J, Stehle E. Effects of postpartum anxiety disorders and depression on maternal self-confidence. Infant Behav Dev. 2012;35(2):264-272.10.1016/j.infbeh.2011.12.005

33. Brockington IF, Oates J, George S, Turner D, Vostanis P, Sullivan M, Loh C, Murdoch C. A Screening Questionnaire for mother-infant bonding disorders. Archives of Women's Mental Health. 2001;3(4):133-140.10.1007/s007370170010

34. Reck C, Klier CM, Pabst K, Stehle E, Steffenelli U, Struben K, Backenstrass M. The German version of the Postpartum Bonding Instrument: Psychometric properties and association with postpartum depression. Archives of Women's Mental Health. 2006;9(5):265-271.10.1007/s00737-006-0144-x

35. Gründahl M, Weiß M, Maier L, Hewig J, Deckert J, Hein G. Construction and validation of a scale to measure loneliness and isolation during social distancing and its effect on mental health 2021. doi:10.31234/osf.io/f7rp6.

36. Narang K, Enninga EAL, Gunaratne M, Ibirogba ER, Trad ATA, Elrefaei A, Theiler RN, Ruano R, Szymanski LM, Chakraborty R, Garovic VD. SARS-CoV-2 Infection and COVID-19 During Pregnancy: A Multidisciplinary Review. Mayo Clin Proc. 2020;95(8):1750-1765.10.1016/j.mayocp.2020.05.011 
37. Almeida M, Shrestha AD, Stojanac D, Miller LJ. The impact of the COVID-19 pandemic on women's mental health. Arch Womens Ment Health. 2020;23(6):741-748.10.1007/s00737-020-01092-2

38. Basu A, Kim HH, Basaldua R, Choi KW, Charron L, Kelsall N, Hernandez-Diaz S, Wyszynski DF, Koenen $\mathrm{KC}$. A cross-national study of factors associated with women's perinatal mental health and wellbeing during the COVID-19 pandemic. PLoS One. 2021;16(4):e0249780.10.1371/journal.pone.0249780

39. Vigod SN, Brown HK, Huang A, Fung K, Barker LC, Hussain-Shamsy N, Wright E, Dennis CL, Grigoriadis S, Gozdyra P, Corsi D, Walker M, Moineddin R. Postpartum mental illness during the COVID-19 pandemic: a population-based, repeated cross-sectional study. Cmaj. 2021;193(23):E835e843.10.1503/cmaj.210151

40. Bu F, Steptoe A, Fancourt D. Who is lonely in lockdown? Cross-cohort analyses of predictors of Ioneliness before and during the COVID-19 pandemic. Public Health. 2020;186:31-

34.10.1016/j.puhe.2020.06.036

41. Milne SJ, Corbett GA, Hehir MP, Lindow SW, Mohan S, Reagu S, Farrell T, O'Connell MP. Effects of isolation on mood and relationships in pregnant women during the covid-19 pandemic. Eur $\mathrm{J}$ Obstet Gynecol Reprod Biol. 2020;252:610-611.10.1016/j.ejogrb.2020.06.009

42. Ayaz R, Hocaoğlu M, Günay T, Yardımcı OD, Turgut A, Karateke A. Anxiety and depression symptoms in the same pregnant women before and during the COVID-19 pandemic. J Perinat Med. 2020;48(9):965-970.10.1515/jpm-2020-0380

43. Wang X, Hegde S, Son C, Keller B, Smith A, Sasangohar F. Investigating Mental Health of US College Students During the COVID-19 Pandemic: Cross-Sectional Survey Study. J Med Internet Res. 2020;22(9):e22817.10.2196/22817

44. Torales J, O'Higgins M, Castaldelli-Maia JM, Ventriglio A. The outbreak of COVID-19 coronavirus and its impact on global mental health. Int J Soc Psychiatry. 2020;66(4):317-

320.10.1177/0020764020915212

45. Mukhtar S. Psychological health during the coronavirus disease 2019 pandemic outbreak. Int J Soc Psychiatry. 2020;66(5):512-516.10.1177/0020764020925835

46. Son C, Hegde S, Smith A, Wang X, Sasangohar F. Effects of COVID-19 on College Students' Mental Health in the United States: Interview Survey Study. J Med Internet Res. 2020;22(9):e21279.10.2196/21279

47. Findlay LC, Arim R, Kohen D. Understanding the Perceived Mental Health of Canadians During the COVID-19 Pandemic. Health Rep. 2020;31(4):22-27.10.25318/82-003-x202000400003-eng

48. Kim P. How stress can influence brain adaptations to motherhood. Front Neuroendocrinol. 2021;60:100875.10.1016/j.yfrne.2020.100875

49. George A, Luz RF, De Tychey C, Thilly N, Spitz E. Anxiety symptoms and coping strategies in the perinatal period. BMC Pregnancy Childbirth. 2013;13:233.10.1186/1471-2393-13-233

50. Barbosa-Leiker C, Smith CL, Crespi EJ, Brooks O, Burduli E, Ranjo S, Carty CL, Hebert LE, Waters SF, Gartstein MA. Stressors, coping, and resources needed during the COVID-19 pandemic in a sample of perinatal women. BMC Pregnancy Childbirth. 2021;21(1):171.10.1186/s12884-021-03665-0 
51. Salfi F, Lauriola M, Amicucci G, Corigliano D, Viselli L, Tempesta D, Ferrara M. Gender-related time course of sleep disturbances and psychological symptoms during the COVID-19 lockdown: A longitudinal study on the Italian population. Neurobiol Stress.

2020;13:100259.10.1016/j.ynstr.2020.100259

52. Saha K, Torous J, Caine ED, De Choudhury M. Psychosocial Effects of the COVID-19 Pandemic: Large-scale Quasi-Experimental Study on Social Media. J Med Internet Res. 2020;22(11):e22600.10.2196/22600

53. Green J, Huberty J, Puzia M, Stecher C. The Effect of Meditation and Physical Activity on the Mental Health Impact of COVID-19-Related Stress and Attention to News Among Mobile App Users in the United States: Cross-sectional Survey. JMIR Ment Health. 2021;8(4):e28479.10.2196/28479

54. Arslan G, Yıldırım M. Meaning-Based Coping and Spirituality During the COVID-19 Pandemic: Mediating Effects on Subjective Well-Being. Front Psychol. 2021;12:646572.10.3389/fpsyg.2021.646572

55. Naghizadeh S, Mirghafourvand M. Relationship of fear of COVID-19 and pregnancy-related quality of life during the COVID-19 pandemic. Arch Psychiatr Nurs. 2021;35(4):364368.10.1016/j.apnu.2021.05.006

56. Lebel C, MacKinnon A, Bagshawe M, Tomfohr-Madsen L, Giesbrecht G. Corrigendum to elevated depression and anxiety symptoms among pregnant individuals during the COVID-19 pandemic journal of affective disorders 277 (2020) 5-13. J Affect Disord. 2021;279:377-

379.10.1016/j.jad.2020.10.012

57. Ahorsu DK, Imani V, Lin CY, Timpka T, Broström A, Updegraff JA, Årestedt K, Griffiths MD, Pakpour AH. Associations Between Fear of COVID-19, Mental Health, and Preventive Behaviours Across Pregnant Women and Husbands: An Actor-Partner Interdependence Modelling. Int J Ment Health Addict. 2020:1-15.10.1007/s11469-020-00340-x

58. Ahmad M, Vismara L. The Psychological Impact of COVID-19 Pandemic on Women's Mental Health during Pregnancy: A Rapid Evidence Review. Int J Environ Res Public Health. 2021;18(13).10.3390/ijerph18137112

59. Gavin NI, Gaynes BN, Lohr KN, Meltzer-Brody S, Gartlehner G, Swinson T. Perinatal depression: a systematic review of prevalence and incidence. Obstet Gynecol. 2005;106(5 Pt 1):10711083.10.1097/01.AOG.0000183597.31630.db

60. Lebel C, MacKinnon A, Bagshawe M, Tomfohr-Madsen L, Giesbrecht G. Elevated depression and anxiety symptoms among pregnant individuals during the COVID-19 pandemic. J Affect Disord. 2020;277:5-13.10.1016/j.jad.2020.07.126

61. Lee DT, Sahota D, Leung TN, Yip AS, Lee FF, Chung TK. Psychological responses of pregnant women to an infectious outbreak: a case-control study of the 2003 SARS outbreak in Hong Kong. J Psychosom Res. 2006;61(5):707-713.10.1016/j.jpsychores.2006.08.005

62. Elsenbruch S, Benson S, Rücke M, Rose M, Dudenhausen J, Pincus-Knackstedt MK, Klapp BF, Arck PC. Social support during pregnancy: effects on maternal depressive symptoms, smoking and 
pregnancy outcome. Hum Reprod. 2007;22(3):869-877.10.1093/humrep/del432

63. Matvienko-Sikar K, Pope J, Cremin A, Carr H, Leitao S, Olander EK, Meaney S. Differences in levels of stress, social support, health behaviours, and stress-reduction strategies for women pregnant before and during the COVID-19 pandemic, and based on phases of pandemic restrictions, in Ireland. Women Birth. 2021;34(5):447-454.10.1016/j.wombi.2020.10.010

64. Meaney S, Leitao S, Olander EK, Pope J, Matvienko-Sikar K. The impact of COVID-19 on pregnant womens' experiences and perceptions of antenatal maternity care, social support, and stressreduction strategies. Women Birth. 2021.10.1016/j.wombi.2021.04.013

65. Daglar G, Nur N. Level of mother-baby bonding and influencing factors during pregnancy and postpartum period. Psychiatr Danub. 2018;30(4):433-440.10.24869/psyd.2018.433

66. Mayopoulos GA, Ein-Dor T, Dishy GA, Nandru R, Chan SJ, Hanley LE, Kaimal AJ, Dekel S. COVID-19 is associated with traumatic childbirth and subsequent mother-infant bonding problems. J Affect Disord. 2021;282:122-125.10.1016/j.jad.2020.12.101

67. Vazquez-Vazquez A, Dib S, Rougeaux E, Wells JC, Fewtrell MS. The impact of the Covid-19 lockdown on the experiences and feeding practices of new mothers in the UK: Preliminary data from the COVID19 New Mum Study. Appetite. 2021;156:104985.10.1016/j.appet.2020.104985

68. Tank AW, Lee Wong D. Peripheral and central effects of circulating catecholamines. Compr Physiol. 2015;5(1):1-15.10.1002/cphy.c140007

69. Cui W, Aida T, Ito H, Kobayashi K, Wada Y, Kato S, Nakano T, Zhu M, Isa K, Kobayashi K, Isa T, Tanaka $\mathrm{K}$, Aizawa H. Dopaminergic Signaling in the Nucleus Accumbens Modulates Stress-Coping Strategies during Inescapable Stress. J Neurosci. 2020;40(38):7241-7254.10.1523/jneurosci.0444-20.2020

70. Jung YH, Kang DH, Jang JH, Park HY, Byun MS, Kwon SJ, Jang GE, Lee US, An SC, Kwon JS. The effects of mind-body training on stress reduction, positive affect, and plasma catecholamines. Neurosci Lett. 2010;479(2):138-142.10.1016/j.neulet.2010.05.048

71. Jung YH, Jang JH, Lee D, Choi Y, Choi SH, Kang DH. Relationships Between Catecholamine Levels and Stress or Intelligence. Neurochem Res. 2019;44(5):1192-1200.10.1007/s11064-019-02762-z

72. Cabib S, Puglisi-Allegra S. The mesoaccumbens dopamine in coping with stress. Neurosci Biobehav Rev. 2012;36(1):79-89.10.1016/j.neubiorev.2011.04.012

73. Dittrich K, Boedeker K, Kluczniok D, Hindi Attar C, Winter SM, Roepke S, Heim C, Herpertz SC, Bermpohl F. Elevated Inflammatory Markers in Women with Remitted Major Depressive Disorder and the Role of Early Life Maltreatment. Brain Behav Immun. 2021.10.1016/j.bbi.2021.07.024

74. Solmi M, Sharma MS, Osimo EF, Fornaro M, Bortolato B, Croatto G, Miola A, Vieta E, Pariante CM, Smith L, Fusar-Poli P, Shin JI, Berk M, Carvalho AF. Peripheral Levels of C-reactive protein, tumor necrosis factor- $a$, interleukin- 6 , and interleukin- $1 \beta$ across the mood spectrum in bipolar disorder: a meta-analysis of mean differences and variability. Brain Behav Immun.

2021.10.1016/j.bbi.2021.07.014

75. Milaneschi Y, Kappelmann N, Ye Z, Lamers F, Moser S, Jones PB, Burgess S, Penninx B, Khandaker GM. Association of inflammation with depression and anxiety: evidence for symptom-specificity and 
potential causality from UK Biobank and NESDA cohorts. Mol Psychiatry. 2021.10.1038/s41380-02101188-w

76. Sluiter JK, Frings-Dresen MH, Meijman TF, van der Beek AJ. Reactivity and recovery from different types of work measured by catecholamines and cortisol: a systematic literature overview. Occup Environ Med. 2000;57(5):298-315.10.1136/oem.57.5.298

77. Hansen AM, Larsen AD, Rugulies R, Garde AH, Knudsen LE. A review of the effect of the psychosocial working environment on physiological changes in blood and urine. Basic Clin Pharmacol Toxicol. 2009;105(2):73-83.10.1111/j.1742-7843.2009.00444.x

\section{Tables}

Due to technical limitations, tables 1 to 6 are only available as a download in the Supplemental Files section.

\section{Figures}




\section{Figure 1}

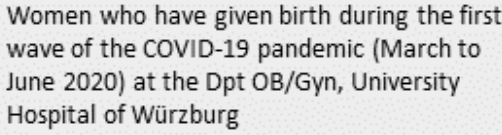

Women who have given birth during the first wave of the COVID-19 pandemic (March to June 2020) at the Dpt OB/Gyn, University Hospital of Würzburg

Throat swab for SARS-CoV-2 PCR analysis and blood collection (serum/plasma)

Questionnaires were filled out by the patients themselves during the delivery stay in the treatment and/or hospital rooms.

- COVID-19 pandemic questionnaire

- Edinburgh Postnatal Depression Scale (EPDS)

- Stress and coping inventory (SCI)

Medical information about course of pregnancy and birth from the patient's record

Samples of placenta, umbilical cord and cord blood, throat swab of the newborn, breast milk swab were collected and frozen
Willingness to participate in the study when admitted to the delivery room?

Met the inclusion criteria?

- age of 18-45 years

- sufficient knowledge of the German language to folllow study information and questionnaires

- Ability to give informed consent

Written informed consent after verbal and written information by the obstetric medical doctors?
Known infection with SARS-CoV-2 during pregnancy?

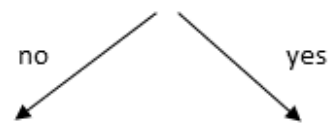

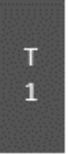

110 patients recruited (April to June 2020)

- 16 patients have withdrawn consent for study participation

$=94$ patients ( 3 patients without biological samples due to the availability of the laboratory)

None

(April to June)

$$
\left[\begin{array}{l}
\text { follow-up } \\
\text { 3-6 months after birth }
\end{array}\right.
$$

62 patients (September to November 2020)

Questionnaires were filled out by the patients themselves at home.

- COVID-19 pandemic questionnaire

- Edinburgh Postnatal Depression Scale (EPDS)

- Stress and Coping Inventory (SCI)

- Anxiety Sensivity Index-3 (ASI-3)

- Parental Bonding Questionnaire (PBQ)

- Lips Maternal Self-Confidence Scale (LMSCS)

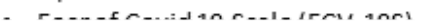

\section{Figure 1}

\section{Supplementary Files}


- GeZeCOcompletedSTROBEcohortchecklist.docx

- GeZeCOTable1211122.pptx

- GeZeCOTable2211122.pptx

- GeZeCOTable3211122.pptx

- GeZeCOTable4211122.pptx

- GeZeCOTable5211122.pptx

- GeZeCOTable6211122.pptx 